A N N A L E S Annales de Bretagne et des Pays de l'Ouest

Anjou. Maine. Poitou-Charente. Touraine

111-1 | 2004

Varia

\title{
Les invitations au banquet d'un homme ordinaire au XXe siècle
}

Arlette Schweitz

\section{OpenEdition}

Journals

Édition électronique

URL : http://journals.openedition.org/abpo/1305

DOI : $10.4000 / a b p o .1305$

ISBN : 978-2-7535-1493-5

ISSN : 2108-6443

Éditeur

Presses universitaires de Rennes

\section{Édition imprimée}

Date de publication : 20 mars 2004

Pagination : $91-110$

ISBN : 978-2-86847-976-1

ISSN : 0399-0826

Référence électronique

Arlette Schweitz, "Les invitations au banquet d'un homme ordinaire au XXe siècle », Annales de Bretagne et des Pays de l'Ouest [En ligne], 111-1 | 2004, mis en ligne le 20 mars 2004, consulté le 19 avril 2019. URL : http://journals.openedition.org/abpo/1305; DOI : 10.4000/abpo.1305 


\title{
Les invitations au banquet d'un homme ordinaire au $\mathrm{xx}^{\mathrm{e}}$ siècle
}

\author{
Arlette SCHWEITZ \\ Ingénieur d'études au CNRS \\ Centre de Recherches historiques
}

Notre intention est de présenter à partir d'une source de type biographique ce que pouvait être le quotidien d'un homme ordinaire, ses invitations au banquet, ses réunions hors de chez lui. Il s'agit en effet d'analyser une centaine de cartes-menus, rassemblés par un marchand de grains entre 1906 et 1970, menus composés par des " traiteurs cuisiniers " de la région chinonaise et de Beuxes, tout en utilisant les notes journalières prises par cet homme. En partant de cette expérience individuelle, il semble possible d'accéder à une meilleure connaissance de la société rurale dans ce qu'elle a de plus banal. Pierre C. est de ceux qui notent minutieusement les faits et gestes de la vie de tous les jours ${ }^{1}$. Il va tenir son journal quotidien à partir de 1928, il a alors trente-sept ans, jusqu'à sa mort. Quelques agendas, conservés par la famille, ont été déposés aux services des archives du Musée national des Arts et Traditions populaires à Paris. Ces premiers carnets ne signalent que très peu de choses. Dans un même temps, il rassemble des coupures de presse, les lettres envoyées à sa famille pendant la Grande Guerre et toutes les cartes-menus des dîners auxquels il était convié et cela très tôt dans le siècle. Des repas pris hors de chez soi, il ne reste le plus souvent que peu de souvenirs, quelques lignes rappelant la composition du repas mais rien sur l'organisation et l'ambiance ${ }^{2}$. C'est donc l'analyse de l'ensemble de ces documents qui va être entrepris ${ }^{3}$.

1. Notre intention est " d'écrire sur les engloutis, les effacés, sans pour autant prétendre porter témoignage ". Alain Corbin en a fait une démonstration magistrale dans son ouvrage Le monde retrouvé de Louis François Pinagot. Sur les traces d'un inconnu 17981876, Paris, Flammarion, 1998, 336 p.

2. FlandRIN, Jean-Louis et CoBBI, Jane (dir.), Tables d'hier, tables d'ailleurs, Paris, Odile Jacob, 1999, 496 p. notamment : BonNAIN, Rolande, "Les campagnes françaises à table ", p. 275-296.

3. Fonds C. : B 34880 175-2 généalogie, 176 1-64 dossiers médicaux, 173 documents administratifs, B 349177 et 179 baux et actes notariés. B 346 carnet de notes 1917-1948, 151-156 et agendas 1906-1928, 140-143, 145, 146. B 345 agendas 1898-1903, livres de comptes 122-125. B 342 livres de raison (1929, 1930, 1931, 1942, 1943, 1944, 1945, 1946, 


\section{L'homme}

« Je m'appelle Pierre C. Je suis né le 30 juillet 1891 à Beuxes dans la Vienne. " C'est ainsi que commence l'histoire d'une vie ordinaire. Il est le petit-fils de Louis G. (10 janvier 1844-1920) et de Louise B. (1850-1926), le fils de Pierre C. (1864-12 novembre 1934), originaire du Morbihan, marchand de grains à La Roberderie, hameau rattaché à Marçay en Indre-etLoire à sa naissance, et de Florine Guérin (1869-1931). Son père effectue des tournées de Beuxes à Marçay, en passant par les hameaux des Forges et de Crues jusque vers $1903^{4}$. À cette époque, ces deux bourgs, distants de neuf kilomètres, rassemblent l'un 189 habitants, l'autre seulement 119. La Roberderie est située à égale distance de l'un et de l'autre.

Pierre C. fils suit les cours de l'école communale de Beuxes. Après avoir obtenu son certificat d'études, il commence à travailler avec son père. Des documents administratifs nous en donnent une description physique précise : "Homme de taille moyenne, aux cheveux châtains, aux yeux noirs, avec un visage rond ${ }^{5}$."

Appartenant à la classe 1911, il est versé du service armé au service auxiliaire en octobre 1912 comme simple soldat de $2^{\mathrm{e}}$ classe au $9^{\mathrm{e}}$ bataillon du $113^{\mathrm{e}}$ de ligne à Romorantin. Mobilisé en août 1914, il occupe les fonctions de garde-magasin à la $25^{\mathrm{e}}$ compagnie stationnée à Chevilly dans le Loiret en 1916. Il ne sera démobilisé qu'en août 1919. Ayant obtenu une permission exceptionnelle, il épouse le 26 novembre 1917 Marie T., née le 29 septembre 1895 à La Lande, commune de Beuxes ${ }^{6}$. De cette union naissent trois enfants : Marie-Thérèse le 13 mai 1919, Pierre le 29 janvier 1923, qui décédera d'un accident automobile le 5 mars 1969 et Marie Bernadette le 21 janvier $1931^{7}$. Il se sépare assez rapidement de son épouse, sans doute vers 1937. Chacun exploite ses biens immeubles propres d'une façon distincte et séparée ${ }^{8}$. Propriétaire et cultivateur, il hérite de l'entreprise de grains familiale, située à « la Roberderie, commune de Marçay par Chinon, gare de Beuxes $^{9}$ ". Son dossier de demande de décoration du Mérite agricole énumère ses principales responsabilités professionnelles et donne un état précis de ses propriétés, comprenant 3 ha de blé, 1 ha $1 / 2$ de vignes, des prairies, des bois, des vaches, des moutons, des chèvres, deux chevaux $^{10}$. Il reçoit la médaille d'argent puis la médaille d'or à l'exposition de Loudun, deux prix d'honneur aux marchés aux veaux. Il est également

1947, 1948, 1949, 1950, 1956; B 343 livres de raison (1958, 1959, 1960, 1964, 1965, 1966, 1968, 1969, 1970, 1971. B 350183 comptes de laiterie B 351184 comptes de boulangerie. B 344, menus (1912-1945).

4. Série de carnets de tournées B 345.

5. Livret militaire et carte d'identité du 6 octobre 1940, Beuxes ATP 80.175.86.

6. Bulletin de mariage, 26 novembre 1897, Beuxes, ATP 80.175.89.

7. Généalogie ATP B 348 80.175.2.

8. Lettre du 30 octobre 1953, adressée au percepteur de Loudun (Vienne) ATP B 348 80.175.80.

9. En-tête d'une lettre de son père du 18 mai 1917, ATP B 348 80.175.79.

10. Documents administratifs, ATP B 34880.173. 
membre du syndicat des agriculteurs de la Vienne ${ }^{11}$, membre du conseil d'administration de la laiterie coopérative de Chinon ${ }^{12}$, président de la caisse locale de Beuxes. Décoré du Mérite agricole par le décret du 15 mars 1930, il devient officier en 1938 sur l'intervention du sénateur de la Vienne Adrien André ${ }^{13}$.

Ses occupations professionnelles l'obligent à se déplacer pour effectuer des livraisons de grains et de vins sur un territoire limitrophe entre la Vienne et l'Indre-et-Loire, notamment à Chinon, Roche-Clermault, Ponçay, Champigny-sur-Veude et Loudun. Dès l'obtention de son permis de conduire en 1937, il abandonne sa carriole pour une Renault qu'il remplacera ensuite par une Citroën ${ }^{14}$.

\section{Les loisirs à la campagne}

Comme l'affirme Jean-Claude Farcy dans son étude sur les loisirs « sans temps libre ", le travail reste un frein à une quelconque évolution et marque toujours la spécificité du monde rural ${ }^{15}$. Cela se vérifie bien au-delà des années trente. La lecture des agendas de Pierre C. nous éclaire sur ses activités. La journée débute toujours par le nettoyage des bêtes et par quelques menus travaux agricoles, dimanche et fêtes comprises. Ce jour, ou plus exactement ce dimanche, est la seule rupture dans une année toute consacrée au travail.

Ses loisirs sont rares et peu nombreux, limités par le temps. Le dimanche d'une fermière dans les Ardennes en 1948 pourrait bien s'appliquer à notre homme qui, rappelons-le, vit seul. " Lever, nourriture des animaux, déjeuner, traite et soins des bêtes, messe (y compris toilette et route), déjeuner hors de chez elle, détente, traite et soins des bêtes, dîner, veillée $^{16}$. "La détente se résume aux invitations entre parents, à la lecture d'un quotidien. L'effet de mécanisation, tant apprécié, ne bouleverse guère cette journée.

11. Le syndicat agricole de Marçay a été créé en 1908, Annuaire statistique et commercial de Touraine.

12. «La laiterie coopérative de Saint-Louans de Chinon, ancienne laiterie coopérative de Chinon, fondée par un groupe de cultivateurs de cette région en 1903. [...] C'est une des plus importantes de la région. Cette laiterie traite les beurres centrifuges extra-fins ainsi que les camemberts dont les principales marques sont le "Chinonais" et le "Bon Ménage". Elle utilise journellement une moyenne de 13500 litres de lait. Elle fabrique également le fromage dit de Sainte-Maure. " La France contemporaine. Grand annuaire, Nantes, impr. du Commerce, 1928-1932.

13. Lui-même est agriculteur, député de la Vienne de 1928 à 1936 puis sénateur de 1936 à 1944. Il est conseiller général du canton de Savin et maire de Béthines dès 1933. Ses interpellations à la Chambre portaient essentiellement sur le commerce du blé.

14. Permis de conduire, ATP B 34880.173.

15. FARCY, Jean-Claude, "Le temps libre au village (1830-1930)", dans CoRBIN, A. (dir.), L'Avènement des loisirs (1850-1960), Paris, Aubier, 1995, p. 274.

16. BECK, Robert, Histoire du dimanche de 1700 à nos jours, Paris, L'Atelier, 1997, p. 344. 
Jusque dans les années quarante, Pierre C. ayant reçu une éducation chrétienne, assiste régulièrement à la messe après s'être occupé des animaux, avoir fait sa toilette et ciré ses chaussures ${ }^{17}$. Ses sorties et divertissements se limitent à peu de choses, " déjeunant chez moi ", sans doute chez ses parents ou avec sa femme dont il se sépare rapidement, souper chez Petit Louis fils après une partie de chasse ${ }^{18}$.

Il participe en tant qu'invité aux grands événements familiaux, communion et mariage, mais c'est le cabaret qui demeure le lieu privilégié de ses loisirs. Il y passe le dimanche après-midi à consommer une chopine et à faire une " coinchée ", une partie de cartes entre voisins, plus rarement à jouer au billard. Il profite de ce déplacement au bourg pour acheter son tabac et ses allumettes, parfois des médicaments ${ }^{19}$. Il lui arrive de chasser et il est abonné à la Dépêche qu'il lit régulièrement ${ }^{20}$.

Les distances parcourues en bicyclette, environ cinq kilomètres séparant la Roberderie de Beuxes, ne sont pas un frein à cette forme de détente. Mais l'après-guerre casse la monotonie de ses loisirs. Tout en débutant toujours la journée de repos par les soins aux bêtes et les travaux de jardinage, il n'assiste plus régulièrement à la messe dominicale mais l'écoute sur son poste de radio. La danse et les bals champêtres, bals de bistrot ou de grange, organisés pour la jeunesse ne conviennent pas à un homme mûr et, semblet-il seul, plus attiré par une sociabilité restreinte et mieux adaptée à son âge, celle des sociétés. Une appartenance de longue date au même terroir, des activités en étroite relation avec la terre lui donnent un sentiment d'appartenance, qu'il n'exprime pas en tant que telle dans ses calepins mais que l'on peut ressentir par sa présence dans les différentes associations, notamment celles des anciens combattants et de la coopérative agricole. Le temps de repos est toujours consacré au nettoyage de la voiture, aux bêtes, aux achats, aux démarches administratives mais s'y ajoutent des réunions syndicales ou professionnelles. Le tout est souvent conclu par un repas.

\section{Les prétextes à se mettre autour d'une table}

Des fêtes et réjouissances passées, il ne reste le plus souvent aucun document. Parfois, un menu dont la lecture, bien des années après, ravive des sensations oubliées. C'est peut-être pour cette raison que Pierre C. a conservé une centaine de cartes-menus entre les années 1906 et $1967^{21}$. Le

17. Cf. une lettre adressée à ses parents en mars 1918, différents documents attestant le versement au denier du culte, des prières transcrites ou découpées dans des journaux, telles que la prière pour la France et les soldats, la Présentation de la Sainte Vierge, et une collection d'images pieuses. Ses relations amicales avec le curé du village de Sammarcolles, situé à cinq kilomètres de Loudun, sont confirmées par des invitations à des déjeuners, par exemple le 24 mai 1932.

18. Agenda 15 sept 1929.

19. Agendas 1929, 1931.

20. Permis de chasse en août 1928. Agendas 1935, 1937, 1938, 1939, 1942.

21. B 344 menus. 
menu marque la place à table de chaque convive, et chacun peut l'emporter chez soi. Il peut être gardé et constitue une collection de souvenirs au même titre que des photographies.

Les prétextes de réunion autour d'un repas sont d'ordre familial et associatif, marqués par la présence d'une carte où s'inscrivent les plats proposés aux convives. Mais fêter un événement ou tout simplement marquer le plaisir d'une rencontre ne donne pas toujours lieu à un support imprimé. Pierre C. a noté d'autres repas dans ses agendas. La diversité des circonstances rend ses documents d'autant plus intéressants, puisqu'il s'agit, pêlemêle, de déjeuner amical, de "repas à l'improviste ", de déjeuner dominical, de banquets privés (communion et mariage), de réveillon mais aussi de banquets de société (des anciens combattants, de la Sainte-Cécile, de la coopérative agricole).

\section{Le déjeuner amical}

Le $\mathrm{xx}^{\mathrm{e}}$ siècle est une période où les jeux d'opposition entre la cuisine de fête et la cuisine ordinaire se trouvent brouillés, non seulement parce que l'on mange de la viande tous les jours, mais encore parce que la structure même du repas quotidien tend à rejoindre, en plus simple, celle du repas de fête.

Pour le remercier de son aide aux travaux des labours, il est invité un dimanche chez Deboine et déjeune d'un potage gras suivi d'un bouillon de poule, de bœuf en sauce, d'un canard rôti et d'une salade. Le tout est accompagné de pain et de foué blanc, de prunes et de gâteaux, de vins blancs et rouges ${ }^{22}$. Quelque dix années plus tard, les quatre heures de repas de battage à la Fénetrie sont suivies d'une invitation à un déjeuner, sans grande recherche gustative : du tapioca, du pâté, suivi de poisson à la mayonnaise, de poulet aux champignons avec des haricots verts, du canard rôti avec salade, d'une salade de fruits, de gâteaux secs, conclu par un café et une eau de vie. Le repas se termine vers 18 heures ${ }^{23}$.

Pour fêter l'anniversaire de Pierre C., le repas subit quelques améliorations au goût de l'invité d'honneur. En 1945, il commence par un œuf dur sauce blanche suivi de veau au beurre accompagné de haricots, d'un lapin, le tout est arrosé de vins rouge et blanc et se termine par une eau de vie ${ }^{24}$.

\section{Le banquet}

À la différence de simples repas, le banquet est annoncé longtemps à l'avance. Il rompt avec l'ordre banal des travaux et des jours. C'est la transgression. Prendre tout son temps pour manger, c'est signifier sans équivoque

22. Agenda 29 novembre 1942.

23. 14 décembre 1958.

24. 29 juillet 1945. L'année suivante, le repas est similaire, le veau est remplacé par un poulet fricassé aux bettes. 
qu'on s'affranchit provisoirement des contraintes de travail. C'est se situer dans l'univers du plaisir reconnu et légitime ${ }^{25}$. Existe-t-il des règles liées à la nature et la quantité des aliments à servir selon le nombre de convives et leur qualité? Des plats raffinés sont-ils proposés pour les circonstances nobles et des produits plus grossiers pour les réunions professionnelles?

\section{Les fêtes marquantes de l'année}

Établir le calendrier du temps libre hors le dimanche revient pour l'essentiel à relever les fêtes religieuses, marquant aussi les principales ruptures de l'année agricole ${ }^{26}$. Lié au calendrier religieux traditionnel, ce type de banquet se place dans le registre familial et strictement intime. De l'activité intense (mai, moissons), au temps ralenti de la Toussaint, des douze jours de Noël au jour de la galette des rois, le tout est ponctué des repas du premier janvier, de Noël, en passant par celui du jeudi de l'Ascension et de la Pentecôte.

Le premier janvier n'est pas forcément un jour de fête et de ripailles. Ce jour peut être consacré à aller à la messe à pied, prendre un apéritif et retourner déjeuner chez soi ${ }^{27}$. Ce peut être aussi le jour de dégustation de mets délicats et rares, par exemple des huîtres que Pierre C. est parti chercher chez M. Vilain ${ }^{28}$. Celles-ci sont préparées à la sauce vinaigrette ou à la sauce verte et constituent l'élément essentiel du réveillon. Mais vers 1960 la journée est consacrée à s'occuper des bêtes et aux menus travaux, écouter la messe, classer des papiers ${ }^{29}$.

Le jour de Noël peut être marqué par un déjeuner pris chez le traiteur tenant le café Boinot, suivi des vêpres. Le menu qu'il commanda en 1942 se compose " d'une douzaine d'huîtres, une escalope de veau sauce tomate, du céleri cuit au beurre, un poulet rôti, salade avec du pain blanc, des œufs au lait au caramel et une bûche de Noël au chocolat, café et eau de vie ". Le plus souvent, le repas est pris en compagnie de Georgette et d'Adrienne. Cette journée n'est pas toujours synonyme de joie et de fête puisqu'en 1969, il la passe seul ${ }^{30}$.

Les repas de la Pentecôte ou de l'Ascension sont des repas " améliorés " avec des fruits de saison, cerises et fraises, un millefeuille ou des biscuits, consommés chez lui. Le "déjeuner à l'improviste chez Boinot " en 1944 le prouve : "Sardines à l'huile, omelette, haricots blancs, salade, gâteaux aux pommes, café. » Il faut varier l'ordinaire sans trop s'en détourner.

25. Prost, Antoine, Les Anciens Combattants et la société française 1914-1939, Paris, FNSP, 1977, vol. Sociologie, p. 196.

26. FARCY, Jean-Claude, art. cit., p. 237.

27. Agenda, $1^{\mathrm{er}}$ janvier 1942.

28. Agenda, 31 décembre 1949.

29. Agenda des $1^{\text {ers }}$ janvier 1958 et 1959 .

30. «Lever, rester à la chambre, pris mes remèdes, tapé le lit, déjeuner, écouter la messe, sorti le fumier, écouter les informations, déjeuner, lu, attendre le souper, coucher ". Agenda 25 décembre 1969. 


\section{Le banquet privé}

Les cartes-menus à caractère familial ne concernent que des personnes ayant des moyens financiers pour les faire imprimer mais le plus souvent ces rencontres ne laissent aucune trace. Fêter le premier jour de l'année, autour du repas de réveillon, est dans une certaine mesure l'occasion de se départir de la sérénité classique et de donner accès à la fantaisie. Dans les repas intimes, le nombre des mets ne semble pas exagéré, et est résolument sacrifié à leur finesse et à leur qualité.

Le tiers des invitations est d'ordre familial, le mariage en constituant l'essentiel. Les fêtes rituelles, comme le baptême, la communion ou le mariage, sont respectées et attendues, rassemblant pour les deux premières une seule et même famille. Le repas du 9 juillet 1944 clôture la cérémonie de baptême de René C., un des proches parents de Pierre C. Il est arrosé de vins locaux " La Roberderie 39 ", " Marçay 29 " ou " La Lande 42 ", montrant l'influence du " pays " dans une telle circonstance. Après un verre de vermouth, c'est la succession classique des mets : hors-d'œuvre, volaille (poulet chasseur et pintade à la broche), viandes (noix de veau financière et gigot pré-salé), haricots verts et salade de saison. Les desserts sont de fabrication familiale comme le laisse penser cette énumération : " La crème anglaise, la galette ménagère, la tarte aux cerises, les gâteaux secs assortis et la corbeille de fruits. " Les vins blancs et les vins rouges sont servis en carafe. Tout le repas reflète la simplicité et l'intimité de l'événement, pas de champagne ou de dragées. La première communion est l'occasion de réunir les membres dispersés de la famille, jusqu'à quarante-quatre personnes le jour de la Pentecôte ${ }^{31}$. Les menus sont rédigés à la main indiquant le nom du communiant mais rarement celui de la cuisinière qui est sans doute la propre mère de l'enfant, ou un membre très proche, tante ou grand-mère. Dans un repas, les rubriques classiques du menu ne sont pas forcément respectées. Il commence avec des hors-d'œuvre de saison, " radis roses au beurre, asperges ", de la charcuterie, suivis du poisson de rivière " anguille de Loire marinière ", " filet de bar sauce verte ". Mais l'entrée peut être un " poulet mascotte " ou " aux champignons ", un " chateaubriand cresson " ou peut être composée de quatre plats distincts " civet de lapin de garenne, gigot d'agneau, veau à la portugaise, poulet à la broche " avec des petits pois à la française et la salade. L'entrée est parfois absente du menu pour entamer directement le relevé ${ }^{32}$. Le repas se poursuit avec une simple crème vanille, ou par une succession de desserts " crème anglaise, pain de Savoie, gâteaux secs ", ou " gâteau de Savoie, œufs à la neige, petits gâteaux secs assortis ". La journée avait débuté par une messe à neuf heures et se termine par les vêpres ${ }^{33}$.

31. Déjeuner de la communion de sa fille Marie-Thérèse C. le 22 juin 1930.

32. Déjeuner de communion de James B. du 7 juin 1931.

33. 25 mai 1947 ou 16 mai 1948. 
L'évolution des formes de sociabilité familiale se ressent-t-elle dans l'organisation des agapes d'un mariage? Le mariage dure une journée entière, avec un déjeuner suivi le soir d'un dîner, coïncidant avec un samedi. L'aide de la cuisinière n'est pas obligatoire pour les communions qui se font entre soi, entre parents, autant sa présence comme tiers médiateur lors d'une noce assure la liaison entre plusieurs groupes étrangers l'un à l'autre ${ }^{34}$. D'après ses agendas, les gestes habituels sont le lever, le déjeuner, le nettoyage de sa voiture, le cirage des chaussures, la toilette et le rasage. Les cérémonies tant civile que religieuse sont suivies d'un " déjeuner " pris dans un café ou une auberge jusque vers $15 \mathrm{~h} 30$. Pierre $\mathrm{C}$. retourne chez lui et revient pour le " dîner ", qui se termine par un bal jusqu'à la nuit très avancée ${ }^{35}$.

La fréquence et la hiérarchie des produits sont étroitement liées au temps de préparation et à la disponibilité selon les saisons. Pour le repas du soir, le potage remplace les hors-d'œuvre variés. L'entrée comprend deux plats (volaille et viande), accompagnés de légumes verts, un rôt avec salade, suivie d'une abondance de mets sucrés, sous forme de petits gâteaux variés, de baba à la crème, bavaroise, et dragées. Le "gâteau de noce ", commandé à un pâtissier, est une pièce montée, à étages superposés, souvent glacée au sucre blanc, soit un nougat monté. Son absence est souvent compensée par une profusion de desserts, de quatre ou cinq sortes. Le sucre est distribué en abondance dans une telle circonstance ${ }^{36}$.

Voici la composition du menu de son mariage le 26 novembre 1917 à Beuxes :

\author{
Potage de tapioca \\ Poisson \\ Bar sauce mousseline \\ Entrées \\ Poulet sauce tartare \\ Filet de bœuf sauce madère \\ Légumes \\ Petits pois à la française \\ rôt \\ Dinde truffée \\ Gigot d'agneau Pré salé \\ Salade de saison \\ Entremets \\ Crème à la vanille \\ Desserts Assortis
}

Vins Rouge et Blanc en carafe. Rouge Launay 1895,

Blanc Ternay 1910. Blanc Marçay 1893. Champagne.

34. Verdier, Yvonne, Façons de dire, façons de faire, Paris, Gallimard, 1979, « La cuisinière" ", p. 261-334, p. 270.

35. Par exemple, le mariage des 8 octobre 1929 (à 2 heures du matin), 30 juin 1930 (à une heure du matin), 22 mai 1944 (à 4 heures du matin), 18 janvier 1947 (à une heure du matin), $1^{\text {er }}$ décembre 1962 (à $2 \mathrm{~h} 30$ ).

36. VAN GenNEP, Arnold, Le Folklore français. Du berceau à la tombe, Paris, Robert Laffont,

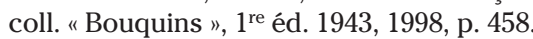


Le dîner est introduit par le potage, et suivi d'un ou deux plats à chaque rubrique. Le repas est arrosé de vins de pays et se termine par du champagne imprimé en gras dans le texte.

En pleine guerre, la gastronomie et les problèmes de ravitaillement ne vont pas de pair. Un arrêté, du ministère des Travaux publics, des Transports et du Ravitaillement, le 25 janvier 1917, publié dans le Journal Officiel, signé Herriot, éclaire notre propos. "Considérant qu'il convient, dans un intérêt patriotique, d'éviter tout gaspillage de produits alimentaires et d'enrayer la hausse des denrées nécessaires à la consommation publique... À partir du 15 février 1917, la consommation des denrées alimentaires dans les hôtels, pensions, restaurants, buffets, wagons-restaurants, cercles, clubs, crémeries, coopératives de consommation, cantines, buvettes et tous établissements de consommation ouverts au public est soumise à une réglementation. Art 1. Il est interdit de servir, à la même personne, un nombre de plats supérieur à deux, dont un seul de viande, un potage ou un hors-d'œuvre (limité à quatre sortes), un fromage ou un dessert (fruits, confiture, compote, marmelade, pâtisseries). Les légumes, cuits ou crus, sont comptés pour un plat quand ils sont consommés séparément, c'est-à-dire quand ils ne servent pas de garniture. Afin de réduire la consommation de la farine, du lait, des œufs et du sucre, l'entremets est supprimé ${ }^{37}$."

Le nombre exact de convives d'un mariage n'est généralement pas connu. Il y a, par exemple, vingt personnes assises à l'une des tables disposées en $\mathrm{T}^{38}$. Le jour du mariage de la fille de Pierre C., Marie-Thérèse, le 2 mars 1946, débute toujours par les gestes habituels : "s'occuper des bêtes, chevaux et bœufs, déjeuner, "relever" le fumier, cirer les chaussures, se préparer et s'habiller, parti à la Lande, assister aux cérémonies civile et religieuse, photographier puis déjeuner de hors-d'œuvre (saucisson, coquilles de beurre et pâté), suivi de canard en salmis, de noix de veau financière, d'un gigot d'agneau et de soissons au beurre, de poulet à la broche, avec en dessert un baba à la crème, une marquise au chocolat et des cafés, le tout arrosé de vins blancs et rouges en carafe ". Le repas commence à 15 heures avec 60 invités et se termine par un bal chez Bodin avec un retour chez lui à 23 heures.

\section{Le banquet entre hommes}

Se grouper pour mieux défendre ses intérêts est sans doute la raison de l'appartenance de Pierre C. à la Coopérative agricole de La RocheClermault et à la Laiterie coopérative de Saint-Louans ${ }^{39}$. Il fait partie de ces hommes qui participent aux instances départementales de sa profession,

37. Andrieu, Pierre, Petite histoire de l'étiquette, Paris, M. Ponsot éd., 1945, p. 104.

38. Dîner du $1^{\mathrm{er}}$ décembre 1962.

39. Cf. un des premiers débats engagés sur cette question : AGUlHon, Maurice et Bodiguel, Maryvonne, Les Associations au village, Bibliothèque des ruralistes, Le Paradou, Actes Sud, 1981, 109 p. 
travaillant parfois hors des limites communales. Sa position à l'intérieur du mouvement associatif est loin d'être négligeable. En tant que membre du bureau de la coopérative de La Roche-Clermault, il participe aux réunions, regroupant une quarantaine de personnes et au banquet qui s'ensuit. Pierre C. consacre un temps considérable à ces réunions, la journée tout entière, celle-ci se terminant généralement à la tombée de la nuit. Elles sont fixées entre novembre et janvier de chaque année. Le repas est organisé à " l'Hôtel Martin " et sa préparation est confiée au " cordon bleu $\mathrm{M}^{\text {lle }}$ Aubert " remplacé en 1956 par l'Hôtel ou le traiteur Stock, et la cuisinière de Cinais, $\mathrm{M}^{\mathrm{me}} \mathrm{L}$. Le nombre des convives dépasse rarement la vingtaine. La composition du repas reflète la simplicité de la carte-menu. Celleci est absente en 1951 et 1952, rédigée à la main en 1950, 1953 et 1954, dactylographiée en 1949 et 1952, imprimée sans aucune recherche esthétique de 1958 à 1961 .

La lecture analytique de ces menus et celle de l'agenda personnel de Pierre C. permettent de définir la hiérarchie des produits proposés à une clientèle rurale et masculine dans la première moitié du siècle. Les indicateurs des préférences gastronomiques sont relevés facilement : horsd'œuvre "variés " ou " choisis" (charcuterie, cœurs d'artichauts, radis, céleri), poisson (brochet, bar, colin ou merlu), entrée unique (poulet, filet de bœuf, veau, paupiette ou noix de veau), poulet et légumes (petits pois, haricots verts) ou dindonneau et légumes, salade de saison, desserts. L'ensemble est arrosé de vins de pays. Les vins de dessert sont le plus souvent offerts par le bureau de la coopérative. Parfois, le repas débute par un apéritif et cela dès 1951. Le " tour de main du cordon bleu ", la tradition et l'économie transparaissent nettement avec l'usage de sauces à préparation rapide, l'omniprésence du poulet rôti accompagné de haricots maître d'hôtel. Seuls les desserts peuvent apporter la petite touche d'originalité.

Pierre C. appartient également au conseil d'administration de la laiterie coopérative de Saint-Louans, dès 1928. Il assiste à l'ensemble des réunions qui se terminent tard dans la matinée et qui sont suivies d'un simple " casse-croûte " vers 14 h $30^{40}$. L'année précédente, il participait à un " casse-croûte " en l'honneur du retour des prisonniers de la laiterie coopérative de Saint-Louans à 14 heures $^{41}$. Ce peut être encore un cassecroûte de "bœuf rôti cornichon et fromage ${ }^{42}$ ", un repas froid " saucisson, pâté, rôti d'agneau, rôti de veau, salade ${ }^{43}$ ". Les deux banquets de la coopérative conservés sont intéressants avec des particularités évidentes tant par les appellations " à la tourangelle ", " de Saint-Louans " que par sa composition $^{44}$ :

40. «[...] été à Chinon, réunion à Saint-Louans, il y avait le casse-croûte mais je n'y suis pas resté. Revenu à 19 h ». Agenda 15 mars 1956.

41. Carte d'entrée pour le casse-croûte du 21 février 1946.

42. 15 juillet 1954 .

43. 20 septembre 1945 .

44. Banquet du 22 avril 1956. 


\author{
Cornet d'York \\ Colin sauce verte \\ Noix de veau Pompadour \\ Petits pois à la Tourangelle \\ Poulet à la Broche \\ Salade de Saison \\ Les Fromages de Saint-Louans \\ Dessert \\ Tarte aux fruits \\ Café - Liqueurs \\ Vin rouge et blanc en carafe
}

Le second menu de la célèbre Hostellerie Gargantua à Chinon ${ }^{45}$, dite la "Charte mensale ", richement décorée d'un énorme Gargantua un verre à la main, déroule tout le plaisir gastronomique chinonais. Il faut alors s'en remettre à la variété et au contraste, et par conséquent à une grande variété des plats et des vins, inconnue en d'autres circonstances. Une connaissance approfondie d'associations savoureuses avec une mise en valeur des denrées sous une forme de cuisson spécifique y transparaît ${ }^{46}$.

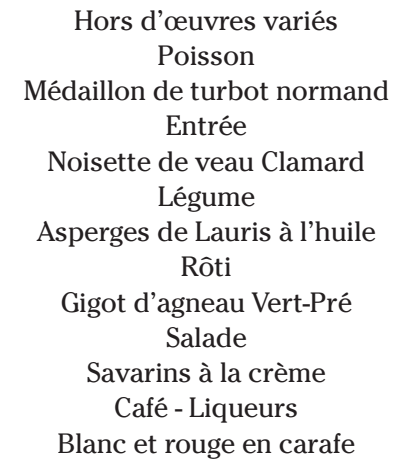

Bien que chasseur, Pierre C. ne semble appartenir à aucune société de chasse. Son adhésion à l'Amicale des Sapeurs-pompiers de Chinon en 1957, ne donne pas lieu à des repas spécifiques. Les banquets de la fête de la Sainte-Cécile, organisés par l'Avenir musical de Beuxes, se déroulent dans le café Bodin. La mise en appétit des convives par des hors-d'œuvre comme le céleri, la charcuterie mais aussi quelques crevettes est suivie par la trilogie poisson (bar ou turbotin), viande (chateaubriand cresson puis selle de veau ou grenadins de veau) et volaille (poulet à la broche). Les haricots verts ou les petits pois accompagnent le plat de subsistance. La salade, dite de saison ou " quelques feuilles à l'huile ", sépare la première partie du repas des entremets (bavaroise au chocolat et biscuits de Savoie) et des

45. Ancien palais du bailliage du XV siècle, tenu par $\mathrm{M}^{\mathrm{me}}$ Casset et propriété de Raoul Dubreuil, antiquaire de Tours, elle est décrite comme " ayant une table copieuse, une cuisine excellente et des mets délicatement préparés. [...] Là en ces murs séculaires, dans un cadre charmant, on est chez soi ". La France contemporaine, 1928-1932.

46. Déjeuner du 24 mai 1939. 
desserts (pithiviers, hollandais, ou corbeille de fruits). Le repas se termine toujours par le café et les liqueurs.

\section{Le banquet d'une " journée combattante "}

Les loisirs au village trouvent leur consécration dans les années vingt puis dans les années trente avec les associations d'anciens combattants. Pierre C., inscrit à l'association des démobilisés de Beuxes « Paix et fraternité ", assiste aux cérémonies commémorant les guerres. L'ordonnance de la journée du 11 novembre se déroule en trois épisodes successifs et bien fixés : un office religieux, une station au monument aux morts et au cimetière, un banquet ${ }^{47}$.

Cette journée n'est pas seulement consacrée au culte des morts pour la France, rassemblant l'ensemble de la communauté. Il s'agit aussi de réunir autour d'un repas les hommes adultes de la commune. De soixantequatorze convives en 1945 à une trentaine en 1946 et 1947, le nombre diminue rapidement pour atteindre une vingtaine dès 1950, et quatorze en 1964 . Le nombre de convives, important pour la commune (près d'un sixième de la population totale), et la présence des autorités contribuent à familiariser le monde rural avec les manières de table bourgeoises. Le banquet peut être précédé d'un vin d'honneur offert par la municipalité ou pris dans un café; il marque le début de la fête. Son importance est soulignée par la présence des autorités locales. Il peut se prolonger par un bal ou par une partie de billard jusqu'à la tombée de la nuit ou l'heure du dîner pris chez soi ${ }^{48}$. La dénomination "Banquet populaire " ou "Banquet amical ", révèle une recherche de cohésion sociale de la communauté masculine plus qu'un repas dédié au souvenir. Le repas est préparé par un traiteur de Beuxes, aidé par une cuisinière ou un cuisinier pâtissier installé en dehors de la commune.

Le menu se présente suivant un ordre immuable malgré la diversité des plats proposés. Il comprend six éléments : " Hors-d'œuvre variés " (céleri, coquilles de beurre, saucisson et pâté - de foie ou de lièvre -), " Poisson " (matelotte d'anguille, merlu - sauce hollandaise, mayonnaise ou à la crème -, carpe farcie, bar - sauce hollandaise ou normande -), "Entrées " (filet de bœuf et veau, filet de bœuf et poulet, gigot d'agneau), "Légumes variés " (petits pois à la française, champignons à la crème, flageolets au beurre, haricots verts, soissons au beurre, asperges sauce mousseline), " Rôt et salade " (poulet à la broche, gigot d'agneau), "Desserts " (gâteaux, corbeille de fruits).

47. Voir à ce sujet, Antoine Prost, op. cit., p. 186 sqq.

48. « Lever, déjeuner, raser, habiller, été avec Georgette aux services des anciens combattants à Beuxes, visites au monument et au cimetière, etc. [sic] été au banquet chez Bodin 400 francs, 30 présents. Fait un billard avec Albert, revenu avec J. Balzeau et Georgette. Retour à 19h30. " Agenda des 11 novembre 1946 et 11 novembre 1948 . 
Les variantes concernent principalement les entrées et les desserts. Les deux viandes, bœuf et veau, la viande et la volaille (bœuf et poulet), sont remplacées dès 1948 par une seule entrée (filet de bœuf financier, noix de veau portugaise ou braisé, veau aux champignons, gigot d'agneau). L'absence de poisson est relevée dès les années soixante ${ }^{49}$. Les desserts comprennent des gâteaux secs assortis, pithiviers, corbeille de fruits, bavaroise chocolat, biscuits de Savoie, marquise au chocolat. Le tout est suivi d'un café et des liqueurs ou d'un café et d'une eau-de-vie.

Le seul " banquet populaire des prisonniers et déportés ", conservé par Pierre C., se distingue de tous les autres par la décoration grivoise de sa carte. Une rangée d'anciens soldats en uniforme et un homme en veston assis devant cinq paires de jambes dansant sur une estrade avec pour légende " quel spectacle magnifique! ». Le repas est préparé par le traiteur de Beuxes et un cuisinier pâtissier de Chinon. Il comprend les rubriques détaillées précédemment, le tout est arrosé de vins de pays ${ }^{50}$ :

\author{
Hors d'œuvre variés \\ Céleri en branches \\ Coquilles de beurre, Pâté Maison \\ Relevé \\ Poulet sauce Périgueux \\ Entrée \\ Noix de veau Portugaise \\ Légumes \\ Haricots verts au beurre \\ Rôti \\ Gigot de Pré-Salé \\ Salade \\ Desserts \\ Génoises aux fruits \\ Gâteaux secs assortis \\ Café Eaux de vie
}

Que ce soit sous la forme d'un banquet, ou plus intimiste, d'un déjeuner, par petites tables, les repas, souvent copieux, servis à ces occasions, sont toujours le prétexte à réaffirmer et à renforcer une convivialité de " clan ".

\title{
Petite histoire du menu
}

L'invention du menu semble être l'un des aboutissements d'un siècle continu d'innovations de 1651 à $1751^{51}$. Il s'écoule un siècle et demi entre

49. Déjeuner amical "Paix et fraternité " du 13 novembre 1960. Déjeuner du 11 novembre 1967.

50. Menu du dimanche 26 août 1945 .

51. Gourarier, Zeev, " Histoire du menu ", La sociabilité à table. Commensalité et convivialité à travers les âges, Rouen, Publications de l'Université de Rouen, $\mathrm{n}^{\circ} 178$, p. 307. Voir aussi PugEt, Henry, " Menus toulousains de la Belle Époque ", L'Auta, n ${ }^{\circ} 275$, juin 1958, p. 81-89 et MoRdaCQ, Philippe, Le menu, une histoire illustrée de 1751 à nos jours, Paris, Laffont, 1989, $154 \mathrm{p}$. 
l'adoption du menu par les classes bourgeoises et son emploi en milieu rural. L'apparition de ces cartes au cours du deuxième tiers du XIX siècle sur les tables alsaciennes semble particulièrement précoce ${ }^{52}$. En Touraine, les premiers menus imprimés semblent apparaître vers 1900. On hésite à enjoliver les menus, en commandant à des artistes la preuve visuelle de ce qu'on veut représenter, en l'honneur d'un événement ou d'une personne. Cela est confirmé par l'étude faite dans le village de Minot : "La vogue des menus, imprimés ou simplement écrits à la main, se développe au village timidement dans les années trente et devient générale et même obligatoire après la dernière guerre ${ }^{53}$."

L'amélioration de l'alimentation des villageois conduit à valoriser la rédaction du menu qui, mieux peut-être que la consommation des plats, fera la différence entre l'ordinaire et l'exceptionnel, entre le déjeuner quotidien et le banquet de noces où les mœurs se libèrent. Dans les campagnes, le terme de menu ne désigne pas un tarif avec une liste de mets ni même une carte de restaurant, mais bien un menu dessiné et préparé pour un repas de fête ou de réunion. Celui-ci laisse deviner au convive la suite du repas et permet d'anticiper dès les premiers mets servis. Il renseigne précisément sur la composition du repas et sur la succession des plats.

Le menu désigne l'ensemble des mets et des boissons qui entrent dans la composition d'un repas. Il s'applique également à la carte, quelles qu'en soient la matière et la forme, sur laquelle le programme est transcrit, et dont un exemplaire est placé avec le couvert, devant chaque convive ${ }^{54}$. Le caractère individuel de la carte met en valeur la générosité de l'invitant et rehausse le faste du banquet. L'improvisation n'est pas de mise. Sont indiqués parfois le traiteur, la cuisinière et l'imprimeur responsables de l'œuvre. La diminution du nombre de mets permet d'inscrire l'ensemble du repas ainsi que le vin sur des cartes de format restreint ( 80 x $150 \mathrm{~mm}$ en moyenne).

\section{Une iconographie soigneusement choisie?}

D'une écriture ronde ou tout simplement imprimé, la lecture du menu participe à la célébration des mariages, des baptêmes, à la commémoration des anniversaires d'une association. Le menu sert de support publicitaire à un hôtel, à une auberge, au traiteur qui l'élabore. Ceux qui ont pu être conservés par Pierre C. sont illustrés (44 sur 50 jusqu'en 1945, et au delà 10 sur 45). La très grande majorité sont imprimés, seulement 17 sur 95 ont été rédigés à la main.

L'intervention de véritables artistes est rare sauf dans le cas d'utilisation de cartes pré-décorées, et par conséquent destinées à toutes sortes d'usages. On y relève fort peu de symboles, quelques initiales entrelacées

52. Lotz, F., Cartes et menus alsaciens, Exposition Musée de Pfaffenhofen, 1978, 14 p.

53. VERDIER, Yvonne, op. cit., p. 277-279.

54. EsCOFFIER, Auguste, Le Livre des menus. Complément indispensable du guide culinaire, Paris, 1915, [rééd. 1996], 193 p., p. 7. 
des fleurs ou entrelacs. La décoration est limitée à celle des mots "menu ", " mariage ", " communion ", parfois s'y ajoutent des fleurs, des oiseaux, généralement dans un angle de la carte. La date n'est jamais omise. Les repas de type organisation professionnelle, en dehors de l'hostellerie Gargantua, sont placés sous le signe de la simplicité. Une distinction est clairement affichée entre les banquets de société et ceux du mariage, ceux du Nouvel an ou de la vie militaire. Ils s'inspirent de l'ambiance facétieuse qui va forcément en découler. L'imprimeur est mentionné; celui-ci est installé à Chinon (F. Moron, Brière, imp. commerciale Saint-Jacques), ou à Loudun (Firmin Blanchard puis Vve Blanchard, Robert, R. Morineau). L'effet de cette inscription est essentiellement commercial.

Toutes ces cartes concernent des circonstances marquantes pour celui qui invite. Pour la plupart d'entre elles, nous connaissons succinctement la cause et la composition du repas. Le plus ancien menu est daté de 1906, c'est un dîner de mariage. La différence entre les banquets du début du siècle et ceux des années cinquante est très visible pour qui observe tous ces bristols. Les couleurs patriotiques agrémentent systématiquement tous les banquets des anciens combattants, du 11 novembre et du 8 mai, jusqu'en 1951. Au-delà, ce sont de simples cartes manuscrites ou dactylographiées. Les cartes entièrement dessinées à la main sont rares et sont également manuscrites. Elles concernent des personnes relativement modestes qui n'ont pas les moyens financiers pour l'imprimer, ou des circonstances d'ordre strictement familial, spécialement la communion solennelle. Le menu de la communion de James B., le 7 juin 1931, d'une écriture malhabile, cherche à exalter la pureté de l'enfance avec sur le coin une " bête à bon dieu " symbolisant le bonheur. Les repas de noces sont annoncés par de grandes cartes célébrant les joies de l'amour avec un couple devant les marches de l'église, des angelots, des cœurs entrelacés, les initiales des futurs mariés. Le menu du réveillon du nouvel an rappelle que c'est l'occasion de faire bombance, il est agrémenté parfois de gravures plus grivoises. Liés au développement de la vie associative de la fin du $\mathrm{XIX}^{\mathrm{e}}$ siècle, les déjeuners de banquet d'association sont parfois enjolivés d'illustrations inventives et originales. Ce peut être un boucher avec son tablier, un couteau à la main poursuivant sa victime, un pauvre canard ${ }^{55}$. En général, la gaîté et l'hédonisme bien présents au cours des repas ne sont pas affichés sur ces cartes. Il convient de montrer une certaine sagesse et non pas d'inciter à des débordements dangereux, voire de l'habiller de références cultivées.

\section{Mystification et facétie des appellations}

Il faut nommer ce qu'on mange. Chacun vient demander à l'organisateur du repas les noms de plats. Ce dernier se différencie des autres en amenant sur les tables un exotisme géographique, comme les œufs moscovites, la

55. Menu du 18 janvier 1947. 
poularde à l'anglaise ${ }^{56}$. Ces mots sont chargés de romantisme, pour les uns, empruntés aux livres de cuisine bourgeoise pour d'autres, parfois il s'agit de véritables néologismes, comme les " grains de fermière " le laissent supposer. Loin de recouvrir des créations culinaires, ces appellations ne semblent être là que pour mieux masquer le caractère récurrent des préparations, ainsi le consommé royal est un bouillon de poule. L'accentuation du caractère mystificateur et facétieux des appellations se généralise après le service de la salade, révélatrice de la division du repas en deux parties ${ }^{57}$. Ce peut être le " Petit Noir et sa Fine " ou le "Petit Noir et son remontant ${ }^{58}$ " ou encore " Grand ordinaire en carafe ${ }^{59}$ ", " le petit Mexicain et sa fine ${ }^{60}$ ", " le Vainqueur du sommeil et son remontant ${ }^{61}$ ". Le banquet de la soixantaine du 14 décembre 1924 est un exemple très exceptionnel, par les dimensions de la carte ( 240 x 155), décorée d'une jeune femme habillée avec soin, portant une corbeille de fruits et un bouquet de fleurs. La dénomination des plats est empreinte de grivoiserie, débutant par un " saucisson à turbulence réduite, suivi des coquilles de beurre (gros colard), un civet de lièvre adulte en rut, de la salade bobine déplumée, des gâteaux avariés ", le tout préparé par $\mathrm{M}^{\text {me }}$ Ravion installée à l'Hôtel du Lion d'or ${ }^{62}$.

\section{Les " organisateurs " ou " les faiseurs " des repas}

La règle est d'entourer l'organisateur ou plus exactement le "faiseur " de repas d'un certain anonymat. Le " chef " dans la rédaction du menu est limité par les prix, par les goûts des clients, par les ressources locales et les propres ressources en personnel. Il est nécessaire d'en faire une rapide description. La lecture de la centaine de cartes menus signale une trentaine de noms de cuisiniers, pâtissiers ou traiteurs, tous localisés dans la zone s'étendant entre Loudun, Chinon et La Roche-Clermault, noms qui sont généralement inscrits en bas du feuillet. Mais il est bien difficile de savoir ce qui sépare le traiteur du cuisinier et du pâtissier. Ainsi le traiteur est autorisé à recevoir une clientèle dans les salles pour y donner à manger, ce qui rend difficile la distinction entre traiteur et aubergiste. On sait que cette cuisine dite populaire est marquée par l'anonymat de ces artisans. Pas ou peu de recettes écrites, et entre les deux, le domaine indéfinissable des tours de mains et des goûts communs, jamais expliqués. C'est treize noms de cuisiniers dont seulement quatre femmes sur un ensemble de 95 menus imprimés, avec la mention du lieu où ils exercent leur métier.

56. VERDIER, Yvonne, op. cit., p. 277.

57. Ibid., p. 278.

58. Déjeuner de la coopérative de La Roche-Clermault, 4 décembre 1960 et 3 décembre 1961.

59. Déjeuner du 9 décembre 1962.

60. Banquet de la coopérative de La Roche-Clermault.

61. Dîner de mariage du $1^{\text {er }}$ décembre 1962.

62. Encore une fois, le repas est préparé par un hôtel restaurant reconnu et dépassant les limites du territoire restreint de Pierre C. 
Les noms de sept " cuisiniers" sont indiqués dans vingt menus. Ces " professionnels " sont installés dans des communes d'importance moyenne, assez éloignées les unes des autres pour ne pas se concurrencer. Il s'agit de Rougeot, (1937), Roy (1950) demeurant à Chinon; Manceau à Loudun (1949), $\mathrm{M}^{\text {lle }}$ Aubert à Roche-Clermault (1950, 1951, 1953, 1954), Lebeaupin à Cinais $(1955,1956,1957,1958,1960,1961,1962)$, Thirot à Vézières (1947) et Martin (1951). Certaines cuisinières sont demandées d'un banquet à l'autre, se spécialisant dans la préparation des repas du 11 novembre ou des réunions de la coopérative. Il y a bien une fidélisation certaine entre la cuisinière et les organisateurs. De 1950 à 1955, les repas de la coopérative sont préparés par M ${ }^{\text {lle }}$ Aubert et de 1955 à 1962 par $\mathrm{M}^{\text {me }}$ Lebeaupin du village de Cinais, indiquée comme lingère dans l'annuaire d'Indre-et-Loire. On s'adresse toujours à des cuisinières résidant dans des villages voisins, leur nom est marqué sur le menu. Elle n'est pas désignée comme " mère " mais comme demoiselle ou comme couple. Figure marginale, parfois célibataire, elle a obtenu une fonction sociale importante et délicate, celle de "faire à manger pour les autres ". Appelée pour les baptêmes, les communions, les mariages, jamais pour les enterrements, c'est aux noces que sa responsabilité est la plus grande.

Le nom de cinq cuisiniers pâtissiers et de deux pâtissiers est indiqué dans 18 repas. Il s'agit d'Opinet (1912), Ragobert de Chinon et cuisinière (1925), Pointereau et cuisinier à Chinon (1925, 1929, 1944), Barre cuisinière à Raslay (1930, 1938, 1944), Hiquet cuisinier pâtissier (1945, 1946),

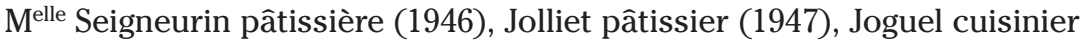
(1949), Bellavoine (1951). La plupart sont associés à un autre " faiseur " de repas. Par exemple Pointereau de Chinon s'associe au traiteur Meunier de Marçay pour un mariage célébré en 1928, ou encore au traiteur Bodin de Beuxes pour le banquet du 26 novembre 1945. Hiquet de Loudun est associé au traiteur Bodin de Beuxes pour le banquet du 11 novembre 1945 ou Meunier de Beuxes le 11 novembre 1946. Si l'aire de rayonnement devient plus large grâce à l'usage de la voiture, on continue de faire appel en majorité à des traiteurs locaux (17 noms sur 55 menus) soit $58 \%$ associés à des cuisinières installées à proximité : Chinon/Marçay - Cinais/La Roche Clermault - Beuxes/Chinon - Beuxes/Loudun.

Pour déguster des plats réellement cuisinés, il convient de s'adresser à certaines bonnes auberges et surtout aux rôtisseurs et traiteurs, rarement mentionnés dans l'annuaire d'Indre-et-Loire (voire les noms en gras) :

- Chinon : Rougeot (cuisinier), Roy (cuisinier), Guiet (traiteur),

Pointereau (cuisinier-pâtissier), Ragobert (cuisinier-pâtissier)

- Loudun : $\mathrm{M}^{\mathrm{me}}$ Manceau (cuisinière), Hiquet (cuisinier-pâtissier), Gilloire (traiteur), Boucher (traiteur), Bigot (traiteur), Opinet (pâtissier) Boesmer (traiteur), Letain;

- La Roche-Clermault : Aubert, M. M ${ }^{\text {me }}$ Stock (traiteurs, tenant le café de la Gare), Mme Brizard (traiteur);

- Raslay : L. Barré (cuisinier pâtissier); 
- Cinais : $\mathrm{M}^{\mathrm{me}}$ Lebeaupin (cuisinière), Thirot, Brizard;

- Marçay (traiteurs) : Meunier (propriétaire du Cercle des bons amis de Marçay en 1924), Pointereau, Plouzeau (spécialisé dans les noces et banquets);

- Beuxes (traiteurs) : Bodin, D. Moreau, Meunier, A. Champigny;

- Azay-le-Rideau : Normand;

- Montcontour : Louis Hulin (cuisinier-traiteur);

- Vézières : M ${ }^{\text {me }}$ Thirot (cuisinière).

\section{De l'auberge à chez soi}

On fait appel à des professionnels qui fixent le prix de leur service en fonction du nombre, du genre et du sexe des convives, et des circonstances qui les motivent. À travers le banquet, c'est l'accession au monde des "bonnes manières ", avec ses nappes, ses serviettes et son code de savoirvivre et de savoir manger que l'on recherche. Les agendas personnels de Pierre C. nous donnent quelques noms sans préciser s'il s'agit de restaurant, de café ou de traiteur. Ceux qui reviennent le plus souvent ont déjà été cités : Boinot, Deboine, et $\mathrm{M}^{\mathrm{me}}$ Garnier à Chinon. Le lieu où se déroule le repas n'apparaît que rarement sur le menu comme si ces repas, entourés de quelques fastes auxquels étaient appelés à prendre part des groupes restreints d'amis ou de membres de la famille pouvaient se dérouler dans une petite auberge ne méritant pas d'être signalée ou tout simplement au domicile privé.

Les organisateurs du repas se sont assuré les services d'une cuisinière, il faut aussi disposer de matériel. Il est possible de commander des repas entiers que l'on se fait servir soit dans une salle louée, soit dans une auberge. Ce " matériel ", utilisé au cours des repas, peut être loué pour la circonstance auprès de trois entreprises, Girault de Loudun, Mitrecé et Meunier. Mitrecé est installé à Beaumont-en-Véron et devient vers 1956 Mitrecé frères ${ }^{63}$. L'entreprise s'agrandit et ouvre une gérance à Loudun vers $1924^{64}$. Elle se situe à cinq kilomètres de Chinon. Meunier, présenté parfois comme traiteur, est installé à Marcay mais semble de moindre importance ${ }^{65}$. Il s'agit pour eux de répondre à une demande exceptionnelle, celle d'un repas de noce. Cela concerne moins de $10 \%$ des banquets.

Bien qu'il ne s'agisse pas encore de restaurants au sens moderne du terme ${ }^{66}$, ces lieux de banquet ont beaucoup de points communs avec les établissements de ce genre. Le terme de " restaurant " dans les annuaires d'Indre-et-Loire apparaît dans des villes de moyenne importance comme Chinon. Il se distingue de l'auberge de campagne comme étant le « temple

63. 13, 14, 16, 17, 24, 29, 85 .

64. 22 .

65. 49, 50 . 
de la haute cuisine ", suivant un statut nouveau de la gastronomie. Mais la fréquentation de ce type d'établissements requiert des revenus substantiels, Pierre C. doit se contenter de lieux plus modestes, où lui et les autres convives allaient prendre un plaisir convivial et gourmand et se donner l'illusion de sortir un peu de leur condition. Il s'agit de se nourrir dans un lieu plus ou moins éloigné de chez soi que l'on associe à ses déplacements professionnels plus qu'à de véritables loisirs, le tout étant facilité par l'acquisition de la voiture. Pour l'essentiel, ce sont des débits de boissons alcoolisées qui consentaient à proposer des plats simples et bon marché, préparés sur place ou apportés depuis une auberge, ou une boutique d'alimentation voisine. Les menus, écrits à la main, gravés ou imprimés, mentionnent rarement l'enseigne d'une hostellerie. Seulement quatre banquets (soit $0,03 \%$ ) semblent avoir été pris dans un restaurant : l'Hôtel du Croissant (1924), l'Hôtel Piet (1967) et l'Hostellerie Gargantua (1939), l'hôtel Moreau à Loudun (1947).

Le menu décrit un repas toujours commandé d'avance, il ne dépasse pas la quarantaine de convives. Le prix est rarement indiqué. Pour payer sa participation au banquet du 11 novembre, Pierre C. débourse en moyenne 400 francs ${ }^{67}$. Pour une communion, il donne soixante francs à la cuisinière. Le service est assuré par le "traiteur " parfois aidé de serveuses. La complexité et la division du travail culinaire sont difficilement discernables. Les tâches les moins nobles semblent souvent abandonnées aux femmes. La composition de l'équipe est rarement indiquée. Pour le repas de la coopérative de La Roche-Clermault, réunissant dix-sept personnes, on comptabilise cinq personnes. Le repas est pris dans le café Stock tenu par le couple, présenté comme traiteurs, la cuisine est assurée par $\mathrm{M}^{\mathrm{me}}$ Lebeaupin et le service assuré par deux serveuses, $\mathrm{M}^{\text {mes }}$ Jean et Marchand ${ }^{68}$.

Les archives familiales - livres de raison, correspondances, documents administratifs, actes notariés -, meurent-elles aussi, non pour avoir trop servies, mais parce qu'elles ne servent plus à rien? En sortant de leur contexte familier, puis de la mémoire de la famille, elles deviennent très rarement des objets d'étude. D'apparence anodine, elles n'ont pas leur place dans la vie de tous les jours, au point qu'une génération oublie de les transmettre à la génération suivante, les dispersent, les brûlent ou les jettent tout simplement; très exceptionnellement, elles sont confiées à des archives départementales ou à des musées. Notre but était d'attirer l'attention sur les questions que soulèvent la conservation et l'exploitation de ce type de document si utile pour l'ethno-historien, permettant ainsi de mieux connaître ce que pouvait être la vie d'un homme "banal ", là où l'exceptionnel n'a pas sa place.

66. PITTE, Jean-Robert, " Naissance et expansion des restaurants ", dans Jean-FLANDRIN, Louis et Montanari, Massimo (dir.), Histoire de l'alimentation, Paris, Fayard, 1996, p. 767-778. 
Aujourd'hui, la commune de Marçay est devenue un lieu du prestige gastronomique, et son château est transformé en un hôtel restaurant, ayant pour chef Pascal Bodin. Il est indiqué dans tous les guides touristiques ${ }^{69}$. Pierre C. aurait-il fréquenté ce type de restaurant?

\section{RESUME}

Inscrite entre une cuisine ordinaire soumise au savoir faire ancestral et une cuisine savante faisant appel à l'invention des cuisiniers, la cuisine des banquets campagnards s'inspire largement des recettes bourgeoises. La centaine de cartes menus, rassemblés par un marchand de grains entre 1906 et 1970 , ont été composés par des " traiteurs cuisiniers " de la région chinonaise et de Beuxes. L'homogénéité de la source offre l'avantage d'une analyse fine de la composition des repas en fonction des prétextes festifs, sur une assez longue période. La somptuosité des banquets, liés à des fêtes familiales, aux réunions de sociétés locales comme la coopérative agricole et la société de sainte Cécile, nous est révélée par ces longues listes de plats mettant en valeur la réalité du patrimoine gastronomique local.

\section{ABSTRACT}

Midway between a common cooking submitted to ancestral skill and a sophisticated cooking requiring the cook' inventiveness, the cooking of the country feasts is highly inspired by bourgeois recipes. The hundred or so menus, collected by a grain trader between 1906 and 1970, were composed by cook-caterers of the region of Chinon and Beuxes. Such homogeneous sources make possible a thorough analysis over a rather long period of how dinners were composed according to the festive opportunities. The long lists of dishes show us how sumptuous the feasts related to family events or to meetings of local societies - such as the agricultural cooperative or the Sainte Cécile society - could be. They also point up the reality of the local gastronomic patrimony.

67. Banquet du 11 novembre : 200 francs (1945), 400 (1946), 500 (1948), 450 (1949), 500 (1950), 800 (1960), 1200 (1967).

68. Banquet du $1^{\text {er }}$ décembre 1957. 